\title{
HALLAZGOS QUIRÚRGICOS EN PACIENTES CON ALOGENOSIS IATROGÉNICA
}

SURGICAL FINDINGS IN PATIENTS WITH IATROGENIC ALLOGENOSIS Dra.Alicia María Tamayo Carbón", Dra. Yiraldi Yanet Bencosme Escarramán", Dr. Rolando Ernesto Medina Robainas 3 .

\section{RESUMEN}

Introducción: Los biopolímeros son macromoléculas cuyo uso como sustancias de relleno con fines estéticos ha ido en aumento en los últimos años. Esto ha generado un incremento de complicaciones por alogenosis iatrogénica de difícil tratamiento.

Objetivo: Describir los hallazgos intraoperatorios en las pacientes con alogenosis iatrogénica intervenidas quirúrgicamente en el Departamento de Cirugía Plástica del Hospital Hermanos Ameijeiras.

Método: Se realizó un estudio descriptivo, prospectivo, longitudinal y monocéntrico con 15 pacientes femeninas que recibieron tratamiento quirúrgico para extracción de sustancias modelantes desde enero 2017 a diciembre 2019.

Resultados: El rango de edad predominante fue entre los 19 y 29 años con un $66.6 \%$ y entre los 30 y 40 años de edad con un $33.3 \%$. El procedimiento quirúrgico fue realizado bajo anestesia general en el $33.3 \%$ de las pacientes; de ellos el $20 \%$ fue en la región mamaria, $40 \%$ glúteos y $40 \%$ genitales y pubis. Se empleó anestesia local en el $66.6 \%$, distribuidas en $20 \%$ en la región frontal y glabelar, $30 \%$ en los párpados y $50 \%$ en los labios. El $100 \%$ mostró hallazgos intraoperatorios similares.

Conclusiones: Independientemente del producto inyectado, la región anatómica y la expresión clínica de la enfermedad, los hallazgos transoperatorios son los mismos. Tanto en las zonas más inyectadas que fueron la región glútea, genital y púbica como en la de menor frecuencia que correspondió a las zonas frontales y glabelar, la extracción del producto resultó en múltiples nódulos en forma de perlas de pequeño tamaño y cavernas.

\section{ABSTRACT}

Introduction: Biopolymers are macromolecules whose use as fillers for aesthetic purposes has been increasing in recent years. This has generated an increase in complications due to iatrogenic alogenosis that is difficult to treat.

Objective: To describe the intraoperative findings in patients with iatrogenic alogenosis who underwent surgery at the Department of Plastic Surgery of the Hermanos Ameijeiras Hospital.

Methods: A descriptive, prospective, longitudinal, single-center study was conducted with 15 female patients who received surgical treatment for removal of modeling substances from January 2017 to December 2019.

Results: The predominant age range was between 19 and 29 years old with $66.6 \%$ and between 30 and 40 years old with $33.3 \%$. The surgical procedure was performed under general anesthesia in $33.3 \%$ of the patients; of them $20 \%$ were in the breast region, $40 \%$ buttocks and $40 \%$ genitalia and pubis. Local anesthesia was used in $66.6 \%$, distributed in $20 \%$ in the frontal and glabellar region, $30 \%$ in the eyelids and $50 \%$ in the lips. $100 \%$ showed similar intraoperative findings.

Conclusions: Regardless of the product injected, the anatomic region and the clinical expression of the disease, the transoperative findings are the same. Both in the most injected areas which were the gluteal, genital and pubic region and in the less frequently injected areas which corresponded to the frontal and glabellar areas, the extraction of the product resulted in multiple nodules in the form of small pearls and caverns.

\section{INTRODUCCIÓN}

os biopolímeros son macromoléculas

cuyo uso como sustancias de relleno con fines estéticos ha ido en aumento en los últimos años. Estos compuestos en la mayoría de los casos son sintéticos, pero pueden ser de origen vegetal o derivados del petróleo ${ }^{1,2}$.

Entre los biopolímeros más utilizados se encuentran las siliconas, constituidas por dióxido de silicio (dimetilsiloxane); el número de enlaces cruzados entre las moléculas de silicio determinan el estado físico de estas, ya sean siliconas fluidas, geles o sólidas. En el siglo pasado la silicona líquida fue muy utilizada, hasta que empezaron a presentarse las complicaciones por el uso de la misma, estos factores en conjunto con los inconvenientes médico especialista de II grado en Cirugía

Plástica y Caumatología, profesor asistente e investigador auxiliar, jefe de servicio Cirugía Plástica y Quemados, Hospital clínico quirúrgico "Hermanos

Ameijeiras". La Habana, Cuba.

2 médico residente de tercer año de Cirugía Plástica y Caumatología. Hospital clínico quirúrgico "Hermanos Ameijeiras". La Habana, Cuba.

3 médico especialista de grado en Cirugía Plástica y Caumatología. Hospital clínico quirúrgico "Hermanos Ameijeiras". La Habana, Cuba.

Correspondencia a:

Nombre: Dra. Yiraldi Yanet Bencosme Escarramán

Correo electrónico:

y_yanet09@hotmail.com

Telf. y celular: +1(809)543-0934 ORCID: https://orcid.org/00000002- 5006-266X

https://orcid.org/0000-00019275-1655

https://orcid.org/0000-00030344-5059

Palabras clave: biopolímeros, rellenos dérmicos, adyuvantes inmunológicos

Keywords: biopolymers, dermal fillers, adjuvants immunologic.

Procedencia y arbitraje: no comisionado, sometido a arbitraje externo.

Recibido para publicación 2 de septiembre 2020 Aceptado para publicación: 5 de septiembre 2020

Citar como:

Tamayo Carbón AM, Bencosme Escarramán YY, Medina Robainas RE. HALLAZGOS QUIRÚRGICOS EN PACIENTES CON ALOGENOSIS IATROGÉNICA. Rev Cient Cienc Med 2021; 24(1): 157-165 
para la correcta interpretación de las técnicas de imágenes y lo difícil del tratamiento, llevaron a la prohibición del uso de la silicona en estado líquido ${ }^{3,4}$.

En el año 2008, el doctor Felipe Coiffman describe por primera vez la enfermedad producida como efecto secundario de la aplicación de estas sustancias y la denominó Alogenosis latrogénica, al estar provocada por el uso de sustancias extrañas al cuerpo a manos del personal de la salud 5 .

La reacción inflamatoria producida por el uso de adyuvantes depende de múltiples factores, que van desde el origen de la sustancia, cantidad y región anatómica infiltrada, hasta los factores propios del paciente que se somete al procedimiento como la hipersensibilidad y la respuesta de los tejidos al compuesto utilizado ${ }^{6}$.

La respuesta autoinmune que se genera tras el uso de modelantes, provoca manifestaciones tanto locales como sistémicas y las características de gravedad del cuadro clínico están determinadas por el tipo de sustancia utilizada. En la mayoría de los casos, los pacientes que se someten a este tipo de procedimiento estético desconocen el compuesto que les es aplicado y se han desarrollado estudios con el objetivo de determinar la sustancia infiltrada, entre ellos el uso de la técnica espectroscópica de resonancia magnética nuclear, la cual ayuda a determinar la estructura química ${ }^{7}$.

Cuando el cuerpo se pone en contacto con sustancias ajenas a él, se inicia una respuesta inmunológica donde se liberan citoquinas tales como Interleukina-1 (IL-1) y Factor de Necrosis Tumoral (TNF) alfa, tanto en la zona tratada como a nivel sérico. A nivel local se produce una fibrosis, debido al acúmulo del biopolímero en el tejido celular subcutáneo, además debido a que estos no son biodegradables puede ocurrir migración del producto en sentido de la gravedad, mayormente en los casos donde se infiltran grandes cantidades del producto ${ }^{8}$.

En el caso de la silicona; una vez que es inyectada produce una reacción celular fibrohistiocitaria, con reclutamiento de células gigantes, que se relaciona con la migración del producto a distancia9.

En el año 2005 Sanz y colaboradores publicaron el caso de un paciente en el que relacionaron la reacción del cuerpo a la silicona con las manifestaciones locales e histopatológicas de un liposarcoma; esto debido al carácter difuso de la reacción con células vacuoladas, histiocitos con configuración irregular, que dan la apariencia de lipoblastos; además la infiltración de tejido muscular vecino, lo que podría interpretarse como un proceso neoplásico ${ }^{10}$.

En respuesta a la aplicación de estos materiales de relleno se producen complicaciones que pueden presentarse de forma inmediata, minutos $u$ horas después del procedimiento, que en la mayoría de los casos son locales, tales como: irregularidades, eritema, discromía, sangrado, equimosis, edema, oclusión arterial y otras manifestaciones que podrían aparecer de forma tardía, meses o incluso años después del procedimiento como: celulitis, dolor, linfedema, nódulos; incluidos en estas la migración a distancia de la sustancia, afectación de órganos y la muerte en el peor de los casos ${ }^{11,12}$.

Nuestra nación no está exenta de ese fenómeno, ya que ha aumentado el número de casos que acuden a consulta en busca de solución quirúrgica para retirarse el producto inyectado ${ }^{13}$.

En los últimos años se ha producido un aumento de la necesidad de mejorar la apariencia física con fines estéticos y con esto ha tenido un gran auge al uso de técnicas no quirúrgicas poco invasivas, pero sobre todo más económicas, con utilización de sustancias no aptas para su uso en el cuerpo, con la finalidad de corregir estas inconformidades de forma rápida, muchas veces en manos de personas no capacitadas. Esto ha provocado un grave problema de salud, debido a los efectos adversos a corto y largo plazo, que requieren un abordaje multidisciplinario para llevar a cabo un tratamiento de larga duración y costoso en la mayoría de los $\operatorname{casos}^{14}$.

Esta enfermedad incurable convierte a quienes la sufren en casos sociales que requieren ser subsidiados por las limitaciones físicas que produce y las deformidades estéticas que ocasiona, lo que los obliga a renunciar a su vida social. Más del $95 \%$ sufren trastornos psiquiátricos y aunque no mueren físicamente si lo hacen mentalmente.

El tema reviste gran importancia científica porque se trata de una enfermedad incurable, cuya solución es la prevención y una vez instaurada solo la extracción del producto modelante mejora los síntomas. Este tratamiento quirúrgico constituye a su vez un 
riesgo de deformidad secundaria y muerte. En los casos en los que la extracción fue muy grande, es muy importante dar seguimiento a los valores de hemoglobina debido al riesgo de hemólisis que experimentan estos pacientes, a causa de la liberación de saponinas, las cuales tienen una alta afinidad por el colesterol de la pared de los hematíes, por lo que es necesario compartir toda experiencia profesional por el bien de los pacientes.

El propósito de este estudio es describir las características de las pacientes que se realizan estos procedimientos, el tipo de anestesia más empleada, las regiones anatómicas más frecuentemente inyectadas y los hallazgos intraoperatorios en el sitio tratado en las pacientes con diagnóstico de enfermedad por modelantes tratadas quirúrgicamente en el servicio de Cirugía Plástica del Hospital Clínico Quirúrgico Hermanos Ameijeiras.

\section{MATERIALES Y MÉTODOS}

Se realizó un estudio, descriptivo, prospectivo, longitudinal, monocéntrico. El universo lo constituyeron las pacientes atendidas en el Servicio de Cirugía Plástica y Caumatología del Hospital Clínico Quirúrgico Hermanos Ameijeiras con el diagnóstico de enfermedad por modelantes que fueron intervenidas para extracción quirúrgica de biopolímeros que cumplieron con los criterios de selección. La muestra estuvo constituida por un total de 15 pacientes femeninas que fueron seleccionadas de forma intencionada en el período de enero 2017 a diciembre 2019. Se incluyeron pacientes mayores de 19 años, con posibilidad de tratamiento quirúrgico que deseaban participar en el estudio, previo consentimiento informado. Se excluyeron las pacientes que no presentaban exposición del material de relleno, fístulas, abscesos o estaba tan disperso y profundo que la cirugía provocaría más daño que beneficio. Para dar respuesta a los objetivos se incluyeron variables como: sexo, edad, tipo de anestesia utilizada (general o local), región anatómica tratada y hallazgos intraoperatorios. Los datos se obtuvieron de las historias clínicas e informes operatorios, se almacenaron en base de datos de Microsoft Office Excel versión XP y se exportaron al sistema SPSS versión 21.0 para su análisis. Los resultados se expresan en porcientos.
Este estudio ha sido realizado según los principios éticos para las investigaciones médicas con sujetos humanos, establecidos en la última actualización de la Declaración de Helsinki (Seúl, Corea, Octubre 2008), la cual se actualizó en Fortaleza Brasil en el año 2013. Los investigadores y todo el personal médico participante en la investigación, poseen experiencia y capacitación para tratar este tipo de pacientes, lo que hizo posible la terapéutica más eficaz y personalizada en cada caso.

Se les garantizó la confidencialidad de la información, como requerimiento de los principios éticos asumidos en el estudio, quedando resguardada y solo fue utilizada con fines de investigaciones o docentes.

\section{TÉCNICAS Y PROCEDIMIENTO}

Preoperatorio: luego de valorar las pacientes en la consulta, y seleccionarlas de acuerdo a los criterios de inclusión, se indicó chequeo preoperatorio y pre-anestésico, se registraron además los datos generales de las mismas. En base al examen físico de cada paciente, en aquellas con deformidades localizadas menores de $5 \mathrm{~cm}$ y posibilidades de cierre borde a borde, se propuso tratamiento quirúrgico con anestesia local y seguimiento ambulatorio. Las pacientes con ulceración, infección secundaria, que requerían la realización de colgajos para el cierre de la zona cruenta, fueron hospitalizadas para tratamiento con anestesia general. Se realizó hemocultivo en casos con síndrome febril y exudado bacteriológico en presencia de supuración.

Procedimiento quirúrgico: luego de realizar asepsia y antisepsia, se colocan paños estériles, se realiza incisión quirúrgica según planificación (previamente diseñada de acuerdo a la localización anatómica de la sustancia modelante, estado de la piel circundante, dimensión de la zona infiltrada y edad de la paciente). Se realiza extracción del tejido dañado, limpieza y cierre por planos previa hemostasia y colocación de drenaje.

Posoperatorio: se indica reposo relativo, no apoyo de la zona quirúrgica, hidratación adecuada, terapia antibiótica, determinación de cifras de hemoglobina posoperatoria, analgesia, curas secas diarias y seguimiento por consulta externa.

RESULTADOS 
El rango de edad predominante fue entre los 19 y 29 años con un $66.6 \%$ y entre los 30 y 40 años de edad con un $33.3 \%$. El procedimiento quirúrgico fue realizado bajo anestesia general en el $33.3 \%$ de las pacientes y anestesia local en el $66.6 \%$. En cuanto a la región anatómica, de las pacientes operadas con anestesia general: el $20 \%$ fue en la región mamaria, $40 \%$ glúteos y $40 \%$ genitales y pubis. De las pacientes operadas con anestesia local: el $20 \%$ fue en la región frontal y glabelar, $30 \%$ en los párpados, $50 \%$ en los labios. Los hallazgos intraoperatorios fueron similares en todos los casos independientemente de la región anatómica y del tipo de sustancia utilizada, encontrándose en el $100 \%$ de los casos un tejido con pérdida de la anatomía normal, con múltiples nódulos en forma de perlas de pequeño tamaño y cavernas con material aceitoso en su interior. (Ver Figura 1, 2 y 3).

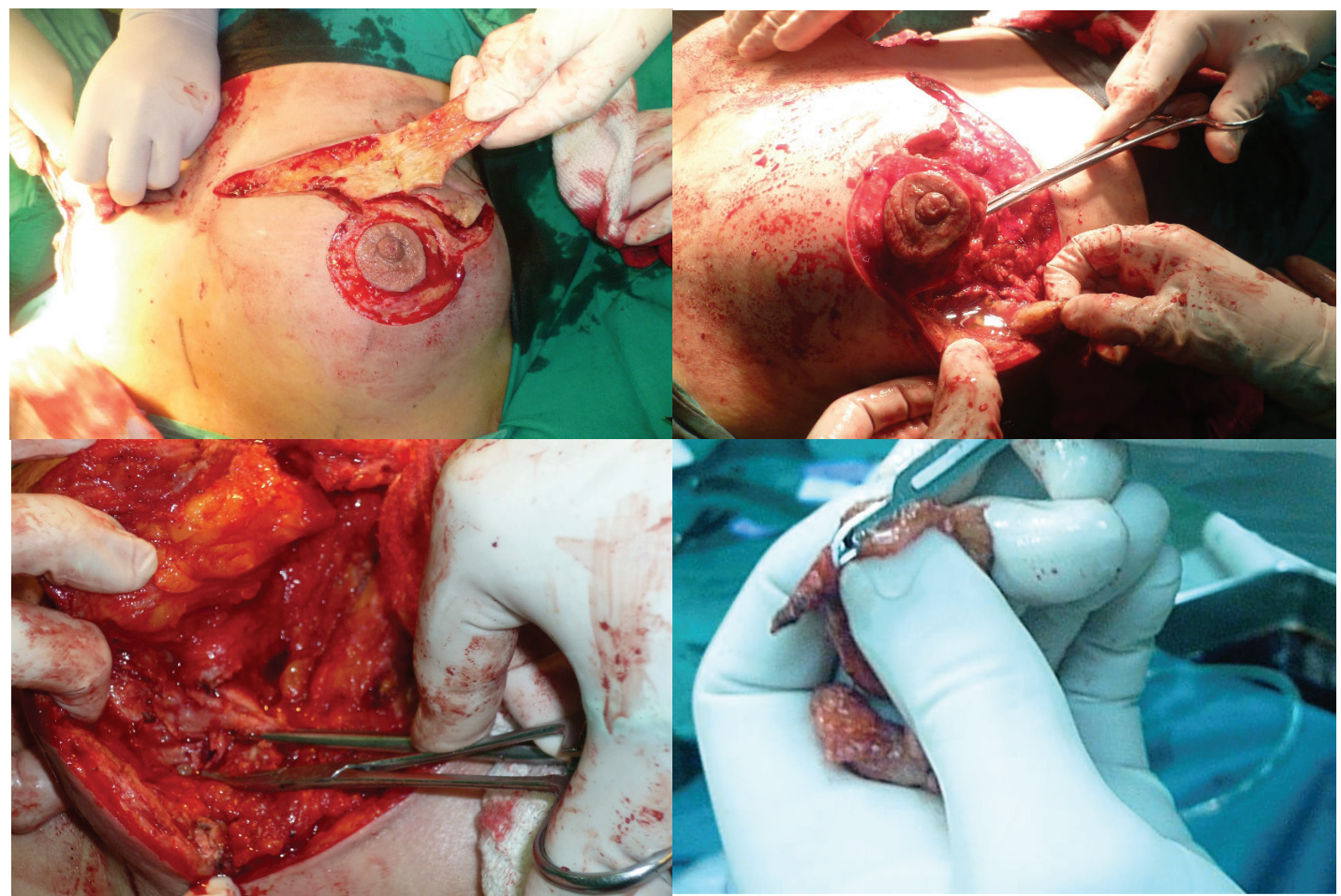

Figura 1. Aspecto macroscópico de la piel y el tejido profundo luego de inyección de sustancia alógena. A: Piel dañada, acartonada. B: Aceite cuando se incide tejido profundo. C: Cavernas y perlas. D: Ruptura de la perla que muestra el contenido derramado en el guante.

Fuente: Fotos del autor.

\section{DISCUSIÓN}

La alogenosis iatrogénica provoca manifestaciones tempranas y tardías que pueden conllevar a secuelas irreversibles, deformidad física y alteración funcional de los órganos afectados ${ }^{15}$.

Las manifestaciones clínicas que se producen con la aplicación de sustancias modelantes, son el resultado de tres fenómenos: la respuesta inmunológica al cuerpo extraño, la migración del producto y la compresión interna que este ejerce en la región anatómica que se encuentra.

La importancia de esto radica en que además de los efectos negativos a la salud y estética del paciente que genera el uso de estos productos, repercute en la calidad de vida y el desarrollo en la sociedad ${ }^{13}$.

El diagnóstico de la enfermedad por modelantes se basa en la sospecha clínica, los antecedentes de exposición, la histopatología y los estudios de imágenes; sin dejar de lado los hallazgos quirúrgicos que son parte importante del diagnóstico definitivo ${ }^{16,17}$.

Esta entidad constituye un grave problema de salud cuyo tratamiento deber ser realizado en conjunto con los clínicos, reumatólogos, patólogos, radiólogos y psiquiatras ${ }^{12}$, debe enfocarse en el alivio de la sintomatología, por medio del uso de antiinflamatorios, antihistamínicos y analgésicos. El tratamiento 


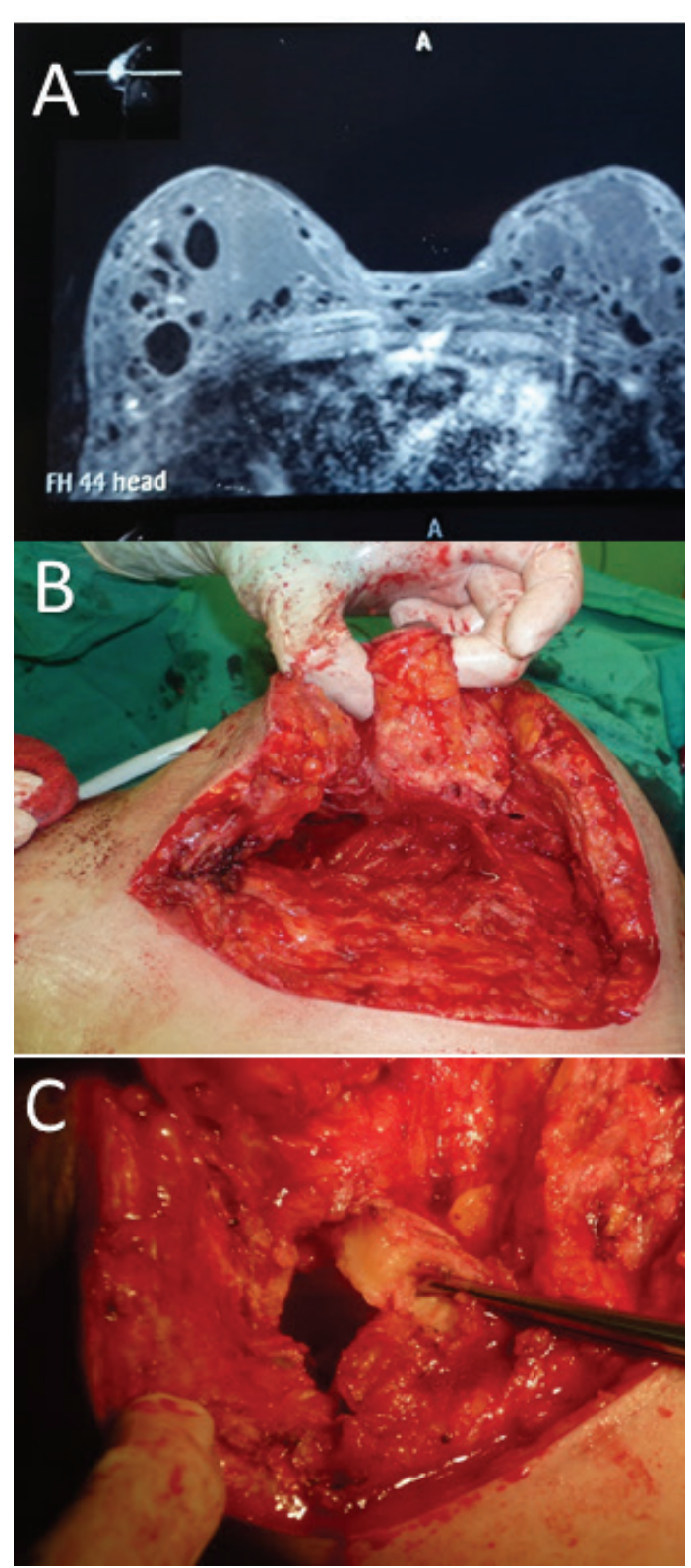

Figura 2. Aspecto macroscópico de tejido mamario con reacción a cuerpo extraño que muestra cavernas visibles por resonancia magnética nuclear. A: Resonancia Magnética. B y C: Cavernas.

Fuente: Fotos del autor.

de elección en pacientes sintomáticos lo constituyen los antiinflamatorios no esteroideos $\left(\right.$ AINES) ${ }^{5}$.

El tratamiento quirúrgico solo se realiza en casos seleccionados dentro de las posibilidades y se basa en la extracción del producto ${ }^{18}$. La dificultad para extraer estas sustancias es una de las razones que hace más difícil el problema, y aún no existe mucha experiencia en este tipo de tratamiento.
En la mayoría de los estudios existe coincidencia con respecto al sexo, en los cuales hay un gran predominio del sexo femenino5, correspondiendo en nuestro estudio al $100 \%$ de los casos.

Estudios como el realizado por Ripalda et al, en pacientes transexuales sometidos a la aplicación de biopolímeros, determinaron que las zonas anatómicas de preferencia son en su mayoría la región glútea y la región mamaria, seguido por la zona facial 19 ; lo que difiere con nuestro estudio en que las áreas tratadas con mayor frecuencia fueron en la región facial.

La estética y los conceptos de belleza tienen un carácter personal y sufre modificaciones a la par del cambio de moda. En nuestro país la mayor tendencia es mostrar labios y glúteos voluminosos lo que influye en los resultados del estudio. El aumento mamario generalmente se realiza mediante la colocación de implantes de gel cohesivo de silicona, pues la mama es un órgano que experimenta modificaciones en el embarazo y siempre existe el temor de trasmitir el producto inyectado en esa zona a través de la lactancia materna; aquí interviene también la edad de la paciente, que en su mayoría son jóvenes.

La respuesta clínica de nuestros pacientes son similares a los casos reportados a nivel mundial, donde los síntomas en su mayoría inician con manifestaciones locales, entre ellas aumento de volumen, edema, eritema; el tiempo de aparición es muy variable, pudiendo ser inmediata hasta años después del procedimiento. Con la investigación se puso de manifiesto que las inyecciones en la región glútea producen manifestaciones con un período de ventana más corto que en el resto de las ubicaciones anatómicas. Así todos los casos con complicaciones que se presentaron a consulta antes del año de la inyección del producto modelante correspondieron a glúteos, el resto presentó complicaciones y deformidades más tardías.

En cuanto al tratamiento quirúrgico la literatura coincide en la dificultad que representa la extracción del producto en un gran porcentaje de las pacientes y se realiza solo en casos seleccionados. En nuestro estudio todas las pacientes que se incluyeron calificaron para recibir tratamiento quirúrgico.

Existe poca literatura que describa las características del tejido encontrado en el acto 

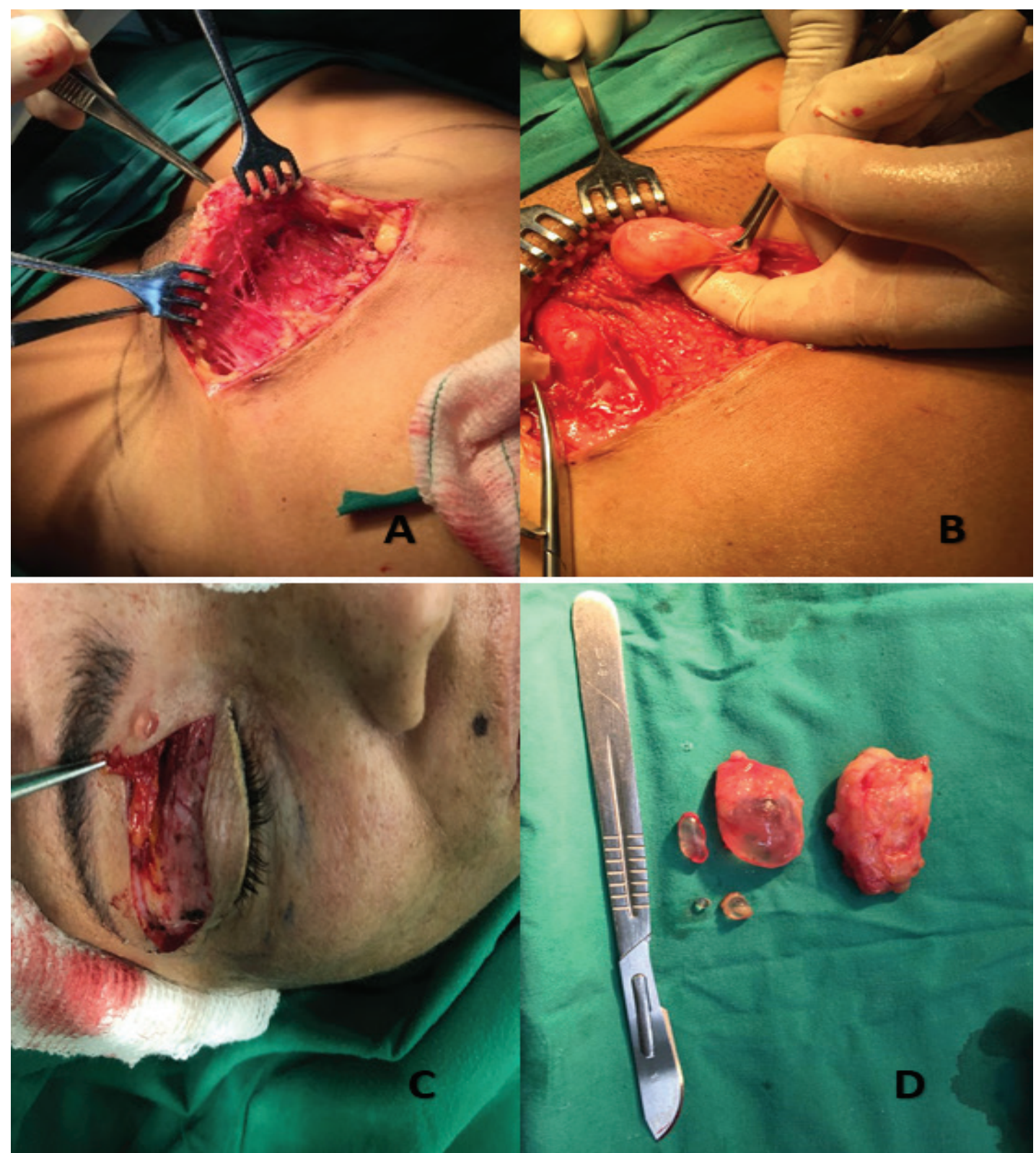

Figura 3. Aspecto macroscópico con formaciones nodulares con aspecto de perlas. A y B: Cavernas y perlas en pubis. C: Perlas en párpados. D: Perlas en genitales.

Fuente: Fotos del autor.

quirúrgico. En nuestro estudio los hallazgos quirúrgicos fueron variables; se encuentra una piel delgada, acartonada y muy adherida al plano profundo, con coloración que varía hasta la más oscura y consistencia hasta la más pétrea. Independientemente de la etiología, tiempo de ventana, cantidad del producto, región anatómica y sintomatología, los hallazgos intraoperatorios fueron los mismos, caracterizados por ausencia de tejido celular subcutáneo en algunas zonas; inmediatamente debajo en el espesor del tejido infiltrado se

abren espacios muertos que dan la impresión de cavernas y que derraman una sustancia aceitosa, clara, con tinte amarillento, inolora, similar al aceite comestible, que recuerdan la imagen de queso Suizo descrito en estudios imagenológicos.

En el tejido se diferencian además múltiples estructuras redondeadas, como perlas de diferentes diámetros, blanquecinas, extendidas en toda el área. Todo esto nos lleva a pensar que los síntomas son expresión de la respuesta inmunológica y no del daño local que genera 
el producto, por lo que no impresiona que hay relación entre los cambios locales y la respuesta inmune sistémica. De esta forma se han presentado casos con marcada afectación local sin otra sintomatología general y otros en contraste con sintomatología florida por respuesta inmunológica sin daño en el sitio de infiltración.

Sulbarán AC et al, publicaron los hallazgos macroscópicos encontrados en tres pacientes con diagnóstico previo de reacción a cuerpo extraño por exposición a material exógeno, cuyo examen físico describe lesiones de aspecto tumoral, con aumento de volumen difuso y fluctuante en la región afectada, asintomáticos. Tras realizar biopsia incisional se encuentran fragmentos redondeados de morfología y bordes irregulares, superficie lisa, brillante, de color blanquecino con tonalidad rosada o blanco-amarillento, de consistencia blanda, tipo glandular, suave, de base sésil y superficie de corte homogénea y delgada22; que guardan similitud con nuestros hallazgos.

En el transoperatorio independientemente de la cantidad o tipo de sustancias utilizadas; de la región anatómica infiltrada, de la magnitud de la respuesta sistémica, y aún en ausencia de manifestaciones locales, en todos los casos se encuentran cavernas y formaciones nodulares esféricas, presencia de material en forma de perlas de diversos tamaños. Las más pequeñas: duras, de consistencia aumentada; las mayores de $5 \mathrm{~mm}$ de consistencia aumentada, pero renitentes y un poco blandas al tacto, que al ser tocadas con el electrocauterio no se desintegran.

\section{CONCLUSIÓN}

A pesar de que desde hace muchos años se conocen las consecuencias del uso de sustancias adyuvantes como relleno, hoy en día sigue en aumento el número de víctimas. El sector de la población que aún continúa exponiéndose a estos procederes son las mujeres jóvenes que debido a esto convierten su futuro en la convalecencia de una enfermedad incurable.

Independientemente del producto inyectado, la región anatómica y la expresión clínica de la enfermedad, los hallazgos transoperatorios son los mismos.

Conflicto de intereses: los autores declaran no tener conflicto de intereses.

Fuente de financiación: los autores declaran no tener ninguna fuente de financiación. 


\section{REFERENCIAS}

1. Sanz Barriga HA, Eróstegui Revilla CP. Alogenosis latrogénica, el gran peligro de los biopolímeros. Rev Cient Cienc Méd [Internet]. 2010 [citado el 6 de abril del 2020]; 13(1):31-34. Disponible en: http://www.scielo.org.6o/scielo.php?script=sci_ arttextsipid $=$ S1817-74332010000100010

2. Domínguez Zambrano A, Haddad Tame JL, Torres Baltazar I, Jiménez Muñoz G, Satré Ortíz N, Espinosa Maceda S. Enfermedad por modelantes: problemática actual en México y presentación de casos. Cir Plást Iberolatinoam [Internet]. 2013 [citado el 6 de abril del 2020]; 39(4):399-405. Disponible en: http://scielo.isciii.es/pdf/cpil/v39n4/original10.pdf

3. Bridges AJ, Vasey FB. Silicone breast implants. History, safety and potential complications. Arch Inter Med [Internet]. 1993 [citado el 3 de abril del 2020]; 153: 2638-44. Disponible en: http://dx.doi. org/10.1001/archinte.1993.00410230048007

Cervera M, Martínez Regueira F, Sola J, Valentí V, Pastor C, Poveda I, et al: Secuelas tras inyección ilegal de silicona líquida como técnica de aumento mamario: Presentación de 2 casos. Cir Esp [Internet]. 2006 [citado el 6 de abril del 2020]; 80(4):227-9. Disponible en: https://www.elsevier.es/es-revista-cirugiaespanola-36-pdf-13093233

4. Coiffman F. Alogenosis iatrogénica: Una nueva enfermedad. Cir Plást Iberolatinoam [Internet]. 2008 [citado el 17 de abril del 2020]; 34(1):1-10. Disponible en: http://scielo.isciii.es/pdf/cpil/v34n1/01.pdf

5. Juárez A, Duran MA, Rivera AM, Tellez S.E, Buitrón $R$, Islas LP. Enfermedad humana por adyuvante en el embazaro. Clin Invest Gin obst [Internet]. 2013 [citado el 15 de abril del 2020]; 40(6): 277-8. Disponible en: https://www.elsevier.es/es-revista-clinica-e-investigacionginecologia-o6stetricia-7-pdf-S0210573\10001103

6. Priego Blancas RB, Jorge Cárdenas R, Pérez Cerezo R, Rincón Lozano R, Torres Gómez B, Luis Haddad J. Enfermedad humana por modelantes. Análisis de sustancias con espectrometría de resonancia magnética. Cir Plast [Internet]. 2010 [citado el 2 de mayo del 2020]; 20(3):120-3. Disponible en: https:// www.medigraphic.com/pdfs/cplast/cp-2010/cp103d.pdf

7. Gutiérrez Gonzalez LA, Pérez Alfonzo R, Párraga de Zoghbi B. latrogenic allogenic case report. Research [Internet]. 2014 [citado el 5 de mayo del 2020]; 1:753. Disponible en: http://dx.doi.org/10.13070/rs.en.1.753

8. Travis WD, Balogh K, Abraham JL. Silicone granulomas: Report of three cases and review of the literature. Hum Pathol [Internet]. 1985 [citado el 2 de mayo del 2020]; (16):19-27. Disponible en: https://doi. org/10.1016/S0046-8177(85)80209-4
9. Sanz ME, Delgado W, Stella Castillo JA, Stella JA. Reacción a la silicona simulando liposarcoma. Folia dermatol [Internet]. 2006 [citado el 20 de abril del 2020]; 17(3):141-4. Disponible en: http://sis6i6.unmsm. edu.pe/bvrevistas/folia/Vol17_NoJ3/pdf/a07v17n3.pdf

10. Murillo Godínez G. Uso illícito de modelantes y efectos adversos. Med Int Mex [Internet]. 2010 [citado el 8 de mayo del 2020];26(4):346-9. Disponible en: https://www.medigraphic.com/pdfs/medintmex/mim-2010/ $\operatorname{mim} 104 \mathrm{~g} \cdot \mathrm{pdf}$

11. Duarte $Y$ Sanchez A, Hedo Toledo AH, Pradel Mora J, Gómez Recilla V. Complicación tardía tras infiltración de biopolímeros en glúteos. Cir Plast Iberolatinoam [Internet]. 2016 [citado el 12 de mayo del 2020]; 42(4):385-9. Disponible en: http://scielo. isciii.es/pdf/cpil/v42n4/reconstructiva4.pdf

12. Tamayo Carbón A, Orozco Jaramillo M, Posada Ruiz D. Adjuvant substances and adjuvant-induced inflammatory autoimmune syndrome. Case presentation. Panorama. Cuba y Salud [Internet]. 2020 [citado el 28 de mayo del 2020]; 15(1):59-64. Disponible en: http://revpanorama.sld.cu/index.php/ panorama/article/view/1120

13. Gordillo Hernández J, Alegre Tamez E, Torres Baltazar I, Mendieta Espinoza MJ, Sastré Ortiz M. Abordaje multidisciplinario de la enfermedad humana por infiltración de sustancias modelantes. Cir plást Iberolatinoam [Internet]. 2013 [citado el 11 de abril del 2020];39(3):269-77. Disponible en: http:// scielo.isciii.es/pdf/cpil/v39n3/original9.pdf

14. Ricaurte Al, Castaño DA, Castro JA, De Paz DA, Echeverry DA. Alogenosis iatrogénica vs. alogenosis secundaria en Cali, Colombia. A proposito de 12 casos. Colomb Forense [Internet]. 2016 [citado el 19 de mayo del 2020]; 3(2):61-72. Disponible en: https:// revistas.ucc.edu.co/index.php/ml/article/view/1778

15. Tamayo Carbòn A, Castellanos Prada A, Aveiro Robalo T. latrogenic alogenosis and rheumatic diseases. Revista Cubana de Reumatología [Internet]. 2018 [citado el 29 de abril del 2020]; 20(2). Disponible en: http://dx.doi.org/10.5281/zenodo.1211650

16. Tamayo Carbón A, Estévez del Toro $M$, Alvarado Salas R, Chong López A. Autoimmune inflammatory syndrome, with symptoms of fibromyalgia, induced by adjuvants after administration of a modelling agent. Revista Colombiana de Reumatologia [Internet]. 2019 [citado el 19 de mayo 2020];26(2):1457. Disponible en: DOI: 10.1016/j.rcreu.2018.02.003

17. Poveda R, Bagán J, Murillo J, Jiménez Y. Reacción granulomatosa facial por rellenos cosméticos inyectados: presentación de cinco casos 
clínicos. Med Oral Pat Oral Cir Bucal [Internet]. 2006 [citado el 12 de abril del 2020]; 11:1-5. Disponible en: http://www.medicinaoral.com/medoralfree01/v11i1/ medoralv11i1p1e.pdf

18. Ripalda A, Loja A, Victor L, Herrera P, J Freites P. Consecuencias clínicas del uso de biopolímeros como prácticas de autoatención en el proceso de transformación corporal en personas transexuales. FACSALUD-UNEMI [Internet]. 2018 [citado el 9 de mayo del 2020];2(2):46-52. Disponible en: http://ojs. unemi.edu.ec/index.php/facsalud-unemi/article/view/723

19. Torres Gómez B, Medrano Ramírez G, Priego Blancas RB, Peláez Ballestas I, Burgos Vargas R. Enfermedad por la infiltración de sustancias modelantes con fines estéticos. Cir Plást.[Internet]. 2010 [citado el 28 de mayo del 2020]; 20(3):124-32.
Disponible en: https://www.medigraphic.com/pdfs/cplast/ cp-2010/cp103e.pdf

20. Ochoa-Pell JA, Escamilla Llano MI. Inyección de materiales extraños para aumento de volumen mamario. Reporte de un caso y revisión de la literatura. Rev Sanid Milit Mex [Internet]. 2006 [citado el 20 de abril del 2020];60(6):417-9. Disponible en: https://www.medigraphic.com/pdfs/sanmil/sm-2006/sm066i. $p d f$

21. Sulbarán AC, Bustillo JA. Datos histopatológicos para identificar una reacción por material de relleno en patologla bucal: revisión bibliográfica y presentación de tres casos. Univ Odontol [Internet]. 2016 [citado el 4 de mayo del 2020];35(74)159-69. Disponible en: http://dx.doi. org/10.11144/Javeriana. uo35-74.dhi 